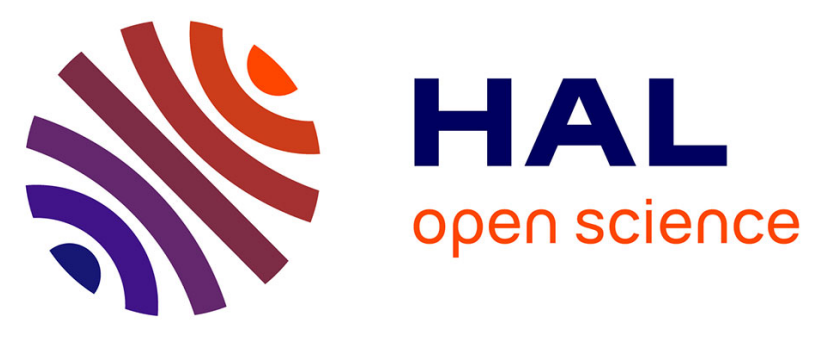

\title{
Vaccination of dogs in an African city interrupts rabies transmission and reduces human exposure
}

Jakob Zinsstag, Monique Lechenne, Mirjam Laager, Rolande Mindekem, Service Naïssengar, Assandi Oussigéré, Kebkiba Bidjeh, Germain Rives, Julie Tessier, Seraphin Madjaninan, et al.

\section{To cite this version:}

Jakob Zinsstag, Monique Lechenne, Mirjam Laager, Rolande Mindekem, Service Naïssengar, et al.. Vaccination of dogs in an African city interrupts rabies transmission and reduces human exposure. Science Translational Medicine, 2017, 9 (421), pp.eaaf6984. 10.1126/scitranslmed.aaf6984 . pasteur03112653

\section{HAL Id: pasteur-03112653}

https://hal-pasteur.archives-ouvertes.fr/pasteur-03112653

Submitted on 17 Jan 2021

HAL is a multi-disciplinary open access archive for the deposit and dissemination of scientific research documents, whether they are published or not. The documents may come from teaching and research institutions in France or abroad, or from public or private research centers.
L'archive ouverte pluridisciplinaire HAL, est destinée au dépôt et à la diffusion de documents scientifiques de niveau recherche, publiés ou non, émanant des établissements d'enseignement et de recherche français ou étrangers, des laboratoires publics ou privés.

\section{(ㅇ)(1) $\$$}

Distributed under a Creative Commons Attribution - NonCommercial - NoDerivatives| 4.0 
2 Vaccination of dogs in an African city interrupts rabies transmission and reduces human exposure

$5 \quad$ Jakob Zinsstag $^{1,2}$, Monique Lechenne $^{1,2}$, Mirjam Laager ${ }^{1,2}$, Rolande Mindekem ${ }^{3}$, Service

6 Naïssengar ${ }^{4}$, Assandi Oussigéré ${ }^{4}$, Kebkiba Bidjeh $^{4}$, Germain Rives ${ }^{1,2}$, Julie Teissier ${ }^{1,2}$, Seraphin

7 Madjaninan $^{3}$, Mahamat Ouagal $^{4}$, Daugla D. Moto ${ }^{3}$, Idriss O. Alfaroukh ${ }^{4}$, Yvonne Muthiani ${ }^{1,2}$,

8 Abdallah Traoré $^{5}$, Jan Hattendorf ${ }^{1,2}$, Anthony Lepelletier ${ }^{6}$, Lauriane Kergoat ${ }^{6}$, Hervé Bourhy ${ }^{6}$, Laurent Dacheux ${ }^{6}$, Tanja Stadler ${ }^{7,8}$ Nakul Chitnis ${ }^{1,2}$

${ }^{1}$ Swiss Tropical and Public Health Institute, PO Box, 4002 Basel, Switzerland

${ }^{2}$ University of Basel, Petersplatz 1, 4003 Basel, Switzerland

${ }^{3}$ Centre de Support en Santé Internationale, BP 972, N’Djaména, Chad

${ }^{4}$ Institut de Recherches en Elevage pour le Développement, BP 433, N’Djaména, Chad

${ }^{5}$ Laboratoire Central Vétérinaire, BP2295, Bamako, Mali

$15{ }^{6}$ Institut Pasteur, Unit Lyssavirus Dynamics and Host Adaptation, WHO Collaborating Centre for

16 Reference and Research on Rabies, 28 Rue du Docteur Roux, 75724 Paris Cedex 15, France

$17{ }^{7}$ Federal Institute of Technology (ETH), Department of Biosystems Science and Engineering,

Mattenstrasse 26, 4058 Basel, Switzerland

${ }^{8}$ Swiss Institute of Bioinformatics, Lausanne, Switzerland 
25 One sentence summary: A citywide dog vaccination effort in Chad reduced the local

26 spread of rabies from dogs to humans.

\section{Abstract}

29 Despite the existence of effective rabies vaccines for dogs, dog transmitted human rabies persists

30 in Africa. Two consecutive dog vaccination campaigns in Chad in 2012 (dog vaccination

31 coverage: $72 \%$ ) and 2013 (coverage: 70\%) interrupted rabies transmission for nine months in

32 N'Djaména, the capital city. We developed a deterministic model of dog-human rabies

33 transmission fitted to weekly incidence data of rabid dogs and exposed human cases in

34 N'Djaména. Our analysis showed that the effective reproductive number, that is, the number of

35 new dogs infected by a rabid dog fell to below one through November 2014. The modeled

36 incidence of human rabies exposure fell to less than one person per million people per year. A

37 phylodynamic estimation of the effective reproductive number from 29 canine rabies virus

38 genetic sequences of the viral N-protein confirmed the results of the deterministic transmission

39 model, implying that rabies transmission was interrupted after the vaccination campaign.

40 However, new dog rabies cases appeared earlier than the transmission and phylodynamic models

41 predicted. This may have been due to the continuous movement of rabies-exposed dogs into

42 N'Djaména from outside the city. Our results show that canine rabies transmission to humans can

43 be interrupted in an African city with currently available dog rabies vaccines, provided the

44 vaccination area includes larger adjacent regions and local communities are informed and

45 engaged. 


\section{Introduction}

48 Dog rabies has been eliminated in large parts of the industrialized countries in Europe and North

49 America. In the last few decades, a concerted effort by South and Central American countries has

50 reduced dog rabies transmission close to elimination (1). Despite the existence of effective

51 vaccines for dogs, dog transmitted human rabies persists and has even re-emerged in Asia and

52 Africa where still more than 59,000 people die annually from this preventable disease. The

53 largest part of the burden is borne by India followed by Africa, China and South East Asian

54 countries (2). Because of rabies' low propensity to transmit secondary infections beyond a bitten

55 individual, it appears feasible to eliminate dog-mediated human rabies through the mass

56 vaccination of dogs $(3,4)$. However, reaching this goal in partnership with the World Health

57 Organization (WHO), the Food and Agriculture Organization of the United Nations (FAO), the

58 World Organization for Animal Health (OIE) and the Global Alliance for Rabies Control

59 (GARC, www.rabiesalliance.org) requires a rigorous scientific approach (5).

61 Reaching sufficient coverage to interrupt dog rabies virus transmission and prevent re-

62 introduction requires an in-depth understanding of dog ecology, dog-human interactions, and the

63 social and cultural determinants of vaccine acceptability, as well as the effective deployment of

64 vaccines with a highly sensitive surveillance system (4, 6-9). It requires scientists to closely

65 collaborate with authorities and communities as partners in a transdisciplinary way between

66 human and animal health $(11,12)$. Concomitant mathematical and economic frameworks can

67 yield new insights into fundamental properties of pathogen transmission (13) and comparative 
cost-effectiveness (14) but do not explain sufficiently how this effectiveness can be achieved

$69(15)$.

71 In 2003, a smallscale study showed the feasibility of dog rabies control in an African city (6) with

72 low cost of US\$ 2-3 per vaccinated dog (16). However, in some African countries, dog owners

73 cannot afford anti-dog rabies inoculations and depend on mass vaccination campaigns that are

74 free of cost $(8,17)$. Analysis of pre- and post-vaccination rabies cases and economic data showed

75 that a single simulated dog vaccination campaign was able to interrupt transmission and was less

76 costly than human post-exposure prophylaxis (14). A proof of the feasibility of dog rabies

77 elimination in an African city would have far reaching consequences for a regionally concerted

78 effort to eliminate rabies in Africa.

80 A city-wide dog rabies mass vaccination campaign was set up in partnership with the Chadian

81 authorities, the Institut de Recherches en Elevage pour le Developpement (IRED), the Centre de

82 Support en Santé Internationale (CSSI) and the Swiss Tropical and Public Health Institute (Swiss

$83 \mathrm{TPH})(23)$. The Chadian government paid for the costs of personnel and logistics and a

84 philanthropic donor paid for the costs of dog vaccines and research. Passive dog rabies and

85 human exposure surveillance started before the campaigns and is still ongoing. Here, we analyse

86 the passive surveillance data of dogs brought to the diagnostic laboratory from this prior work

87 using mathematical transmission models and phylodynamic analyses of dog-related rabies virus.

88 We investigate the impact of the vaccination campaigns for interrupting transmission and the

89 potential for maintaining elimination.

90 


\section{$91 \quad$ Results}

\section{Mass vaccination and field data}

94 The vaccination campaign operations are described in detail in a previous publication (23) and

95 summarized in the materials and methods. Vaccination coverage surveys followed each sequence

96 to assess the achieved coverage and the deficit to reach 70\% target coverage (23). In 2012, $72 \%$

97 of all dogs were vaccinated (95\% confidence interval 69-76\%) and in $201370 \%$ were vaccinated

98 (95\% confidence interval 69-75\%).

100 Our results are based on data obtained about the weekly incidence of dogs newly infected with

101 rabies virus(Fig. 1A) and the incidence of related human exposure (Fig. 1B). These data were

102 collected through passive surveillance, i.e. by suspected dogs that were brought for testing to the

103 rabies laboratory in N'Djaména and through collection of rabies virus isolates from rabid dogs

104 throughout the vaccination campaign, described above. Recorded numbers of vaccinated dogs

105 were used for the estimation of vaccination coverage (23). These data were used to estimate the

106 effective reproductive number $\mathrm{R}_{\mathrm{e}}$ (the number of new rabid dogs infected by one rabid dog at any

107 time, accounting for immunity and interventions, estimated from the transmission model) and the

108 threshold population density of susceptible dogs using mathematical models. The data suggested

109 that mass dog vaccination campaigns in 2012 and 2013 reached sufficient coverage to interrupt

110 rabies transmission from January 2014 to October 2014. Dog rabies incidence in the city of

111 N’Djaména, estimated from passive surveillance, dropped from 0.33 rabid dogs / (10 000*week)

112 prior to the mass vaccination campaign to 0.016 rabid dogs / (10 $000 *$ week) in 2014 (Fig. 1A).

113 Similarly, the incidence of human exposure to rabid dogs, estimated from passive surveillance, 
114 dropped from one human exposed to rabies virus / (1 $000000 *$ week) prior to the mass

115 vaccination to less than 0.002 / (1 $000000^{*}$ week) in 2014, which is less than one person per year 116 (Fig. 1B).

\section{Transmission model}

119 We used a deterministic, population-based model of ordinary differential equations to model

120 rabies virus transmission amongst dogs as well as between dogs and humans (Table S1). The

121 transmission model showed that between the two campaigns in 2012 and 2013, effective recorded

122 vaccination coverage decreased from a peak of 67\% (December 2012) (23) to a trough of 33\%

123 (October 2013), assuming an exponential distribution for the persistence of immunity, which was

124 estimated from 105 immunized dogs undergoing repeated serological measurements. This

125 represents a 51\% relative coverage loss (Fig. 2A). The model suggested that population

126 replacement by the birth of susceptible dogs accounted for $29 \%$ of the relative coverage loss,

127 whereas individual dog immunity loss accounted for $22 \%$ of this relative coverage loss.

129 The effectively vaccinated surface area in our campaign of $240 \mathrm{~km}^{2}$ (2012) was much lower than

130 the $770 \mathrm{~km}^{2}$ assumed in an earlier simulation (14). The empirical data from this study provides a

131 better estimate of parameter values, the threshold density of susceptible dogs and the basic

132 reproductive number, i.e. the number of secondary infections resulting from a typical case in a

133 completely susceptible population, as $R_{0}=1.14$, instead of $R_{0}=1.01$. This means that rabies is

134 more infectious in N'Djaména, than previously reported (14). The effective reproductive number,

$135 \mathrm{R}_{\mathrm{e}}$, decreased from the equilibrium value of 1 from the start of the first vaccination campaign and

136 remained below 1 through November 2014, implying that the conditions for rabies virus

137 persistence were not maintained since the start of the vaccination campaigns. Simulations of a 
138 deterministic ordinary differential equation model (Fig. 1), fitted to rabies case data from

139 N'Djaména, and a stochastic extension (Fig. 3) suggested that rabies transmission was interrupted

140 from early 2013 onwards.

142 As our model did not include importation of infections, we wondered whether dog rabies cases

143 seen from the October 2014 onwards (Fig. 1A) were due to imported cases (with subsequent

144 local transmission) rather than sustained ongoing transmission from the end of 2013 or the

145 beginning of 2014. To test this hypothesis, we performed a maximum likelihood phylogeny of

146 nucleoprotein sequences from rabies virus isolates collected in Chad (from N'Djaména and other

147 regions) from August 2011 to January 2015. Indeed, the dog rabies cases from 2014 onwards

148 were phylogenetically distinct from those previously circulating in N'Djaména (Fig. 4)). We

149 therefore suspected that domestic dogs from surrounding peri-urban and rural areas were the

150 more likely source of reinfection rather than ongoing transmission in dogs or wild animals.

151 Consistent with this, only dog-related rabies virus strains and no wildlife-related strains were

152 found in a previous rabies virus phylogenetic analysis performed in N'Djaména (19).

\section{Sensitivity analysis}

156 We performed sensitivity analysis to determine whether the simulation results were robust

157 compared to our estimates of parameter values. Figure S1 shows simulation results of the density

158 of infectious dogs over 6 years allowing for uncertainty in each of the parameter values (varied

159 one at a time). In each simulation run, the dog transmission rate, $\beta_{\mathrm{dd}}$, was refitted for that set of

160 parameter values. The results were robust to uncertainty in the parameter values and except for

161 low vaccine efficacy values, the simulations predicted that transmission would be interrupted 
162 after the first campaign. Fig. S2 shows a similar sensitivity analysis of the simulated number of

163 infectious dogs but with a fixed value for the dog transmission rate, $\beta_{d d}$, estimated from the

164 baseline set of parameter values (tableTS2). The ranges of the parameter values were greater than

165 in Fig. S1 and the results showed the importance of that parameter on the expected number of

166 rabid dogs over time. Most parameters had little effect, but similar to the sensitivity analysis for

$167 \mathrm{R}_{\mathrm{c}}$, high values for the carrying capacity of dogs and the probability of an exposed dog

168 developing rabies and low values for the rabies induced death rate led to a high number of

169 infectious dogs.

170

171 Fig. S3 shows the simulated densities of infectious dogs and exposed humans depending on the

172 probability of detection of infectious dogs, $p_{d}$, and of exposed humans, $p_{h}$, used to fit $\beta_{d d}$ and

$173 \beta_{\text {hd }}$, respectively. Low values of these detection probabilities result in higher numbers of

174 infectious dogs and exposed humans, leading to higher estimates for the dog to dog, $\beta_{\mathrm{dd}}$, and dog

175 to human, $\beta_{\mathrm{hd}}$, transmission parameters. The results indicated that the simulation data were

176 robust regarding these detection probabilities unless the probabilities were very low and that

177 underreporting of rabies cases was unlikely to have a substantial effect on our results (Fig. S 3).

178 This in turn suggested that underreporting of cases did not play a large role in the persistence of

179 rabies transmission. Even accounting for heterogeneity in underreporting, it was unlikely that

180 unreported transmission persisted for nine months and more likely that a re-introduction

181 occurred, either from wildlife or from dogs with ongoing transmission outside the city of

182 N’Djaména.

\section{Phylodynamic analysis}

18429 nucleotide sequences encoding the N-protein from dog related rabies virus isolates collected

185 between August 2011 and June 2013 were sequenced as previously described (9, 24). Although 
186 the $95 \%$ highest posterior density intervals were wide because of the small number of sequences,

187 the effective reproductive number estimated from the genetic data showed the same pattern as

188 the $\mathrm{R}_{\mathrm{e}}$ estimated from the incidence data (Fig. 2B). We demonstrate thus by two different

189 methods, transmission modelling and a phylodynamic analysis that dog rabies transmission can

190 be interrupted by the mass vaccination of dogs in N’Djaména.

191

192 Discussion

193 The models and data presented here show that the period with no rabies transmission in

194 N'Djaména was longer after mass vaccination campaigns than in the absence of such campaigns,

195 suggesting that dog rabies virus transmission in this African city could be interrupted and

196 consequently human rabies exposure reduced. However, the duration of interruption of

197 transmission in our study was shorter than our model predictions presented here and in earlier

198 work (14), indicating that there was likely to be a re-introduction of infection from wildlife or

199 latently infected dogs from the adjacent areas, similar to what was reported in a recent study on

200 rabies transmission in Bangui (26).

201

202 Our study suggested that urban centres may not be hotspots of dog rabies transmission leading to

203 spill over cases in rural areas, as previously thought. Dog rabies transmission is ongoing in peri-

204 urban and rural African areas and is likely to be continuously transmitted into urban areas

205 through human-mediated transport of dogs (9). Sustainable elimination of dog rabies therefore

206 will require action over a much larger geographical area. We have proposed a development

207 impact bond financing scheme for dog rabies elimination in the entire country of Chad (27). 
209 There is still considerable uncertainty surrounding the role of density and spatial heterogeneity

210 and external re-introduction in the transmission of dog rabies (28). A meta-population or contact

211 network modeling approach may better represent the observed heterogeneity of the dog

212 population in N'Djaména (Fig. S4). Further research is needed to assess how dog density and the

213 spatial heterogeneity of dog populations influences the dynamics of dog rabies elimination (28,

214 29).

215

216 Determining the optimal timing of vaccination campaigns in N'Djaména to maintain elimination

217 would require better knowledge of the rabies importation rate into the city. Our results are in line

218 with a recent study from Bangui, Central African Republic showing that rabies is continuously

219 re-introduced in towns by human related transport of dogs from surrounding peri-urban and rural

220 areas (26) and rapidly dispersed between cities (9). Therefore, we suggest that dog rabies control

221 in African cities should be planned for larger areas, including suburban and rural areas, and be

222 coordinated regionally between neighboring countries for effective elimination of dog rabies in

223 Africa (1). In particular, movement of dogs with or without their owners should be restricted to

224 limit the rapid dispersal of dog associated rabies virus. Dog mass vaccination campaigns should

225 also be complemented by affordable compulsory dog registration. Our study further supports the

226 need for an improvement and a reinforcement of rabies surveillance in rural and more remote

227 areas to achieve inclusive and comprehensive rabies reporting that can then be used to guide

228 vaccination decisions. New rapid tests for rabies could be used in a decentralized manner and

229 may enable collection of data about rabies epidemiology in remote locations (30).

230

231 In contrast to previous reports (31), our study suggests that mass vaccination of dogs, coupled

232 with post-exposure prophylaxis, could be sufficient to eliminate rabies transmission in an 
233 African city, both in dogs and humans, as long as vaccination is extended to a larger area beyond

234 the city itself. In the long term, eliminating the infectious rabies reservoir in dogs will be more

235 cost-effective than perpetual post or pre exposure prophylaxis in humans(32). Dog vaccination

236 campaigns will require a regional approach similar to the well-coordinated dog rabies control

237 efforts among Latin American countries (1). The recent creation of the Pan African Rabies

238 Control Network (PARACON, paracon.rabiesalliance.org) is an important first step towards the

239 goal of eliminating dog rabies from Africa by 2030.

\section{Supplementary materials}

243 Supplementary Figures

244 Supplementary Figure 1: 4 One -dimensional sensitivity analysis of simulation results on

245 parameter values. The plots show simulations of the density of infectious dogs over 6 years (300

246 weeks) where all parameters are fixed at values described in table S2 except for the parameter

247 being varied and $\beta d d$. The $x$-axis shows the time in weeks and $y$-axis shows the value of the

248 parameter (in its corresponding units). The colour of each pixel represents the density of

249 infectious dogs. The horizontal red lines correspond to the parameter values in table S2 and the

250 solution plotted in Fig. 1A and Fig. 2A.

251

252 Supplementary Figure 2: One-dimensional sensitivity analysis of simulation results on parameter

253 values. The plots show simulations of the density of infectious dogs over 6 years (300 weeks)

254 where all parameters are fixed at values described in Table 3 except for the

255 parameter being varied ( $\beta$ dd is fixed at 0.0292). The $\mathrm{x}$-axis shows the time in weeks and $y-$ 
256 axis shows the value of the parameter (in its corresponding units). The colour of each pixel

257 represents the density of infectious dogs.

259 Supplementary Figure. 3: Sensitivity analysis of the simulation results on the probability of 260 detecting rabid dogs. (A) The simulated density of infectious dogs depending on the detection

261 probability of rabid dogs, p_d, over time. The $\mathrm{x}$-axis corresponds to time (measured in weeks)

262 and the y-axis to the detection probability. The colour of each pixel corresponds to the density of

263 infectious dogs. (B) The endemic equilibrium value for density of infectious dogs (in the absence

264 of vaccination campaigns) depending on p_d. The $\mathrm{x}$-axis corresponds to to the detection

265 probability, p_d and the $y$-axis (and colour of the pixel) correspond to the endemic equilibrium

266 density of infectious dogs.

268 Supplementary Figure. 4: Density of vaccinated dogs in N'Djaména in 2013 calculated based on

269 the data presented in Léchenne et al. 2016. Black dots indicate the locations of the fixed

270 vaccination posts. It is assumed that dogs diffuse from these locations after vaccination in a

271 homogeneous way. We used a diffusion kernel prediction map with a bandwidth of $1040 \mathrm{~m}$

272 (which is the diameter of a circle of $0.86 \mathrm{~km} 2$, the area per post of 331 posts in a total area of

$273285 \mathrm{~km} 2)$. The water surface was included as a barrier function.

276 Supplementary Figure. 5: Schematic of mathematical model of rabies. Birth and death rates of 277 humans and dogs are not shown. 
279 Supplementary Figure 6: Vaccination rates during the two campaigns in N'Djaména, Chad a) in

2802012 and b) in 2013. The weeks are labelled starting from 4 June 2012.

281

282 Supplementary Figure 7: Local and global sensitivity indices of the control reproductive number, 283 R_c, to the model parameters.

285 Supplementary Figure 8: Sample simulation of the stochastic model showing the density of 286 exposed and infectious dogs with the simulation results of the deterministic model and the 287 observed number of infectious dogs.

289 Supplementary Figure 9: Results of the phylodynamic analysis showing median (red) and 95\%

290 HPD interval (black) for R_e through time. Solid lines correspond to the constant sampling 291 proportion assumption, and dashed lines to the changing sampling proportion assumption. Blue 292 points indicate the change of R_e and sampling proportion. We plot the R_e estimate for each 293 interval at the midpoint of the interval, and interpolate linearly in between.

Supplementary Tables

296 Supplementary Table 1: State variables of dog rabies transmission (model)

298 Supplementary Table 2. Parameters of the rabies transmission model with estimated values and 299 sources. Most parameters have the same value as in the previous model (14), but some have been 300 updated from more recent publications or from new data from the current study (as described in 301 the section on parameter estimation). 
303 Data files

304 - RabiesData1.txt (incidence data)

305 - RabiesData2.txt (genetic sequences of 33 dog rabies virus N-protein deposited in GenBank)

\section{Materials and Methods}

\section{Study design}

314 The objective of this study was to test the hypotheses a) that dog rabies virus transmission in an

315 African city can be interrupted by the mass vaccination of dogs and $b$ ) that cities are hotspots of

316 dog rabies virus transmission and that re-introduction would be slow. As we observed the re-

317 introduction of dog rabies after the mass vaccination of dogs, we hypothesized that rabies virus

318 was re-introduced from the outside of the city. The research subjects were the weekly number

319 of routinely recorded rabies suspected dogs and the number of exposed humans per rabid dog.

320 The design of the present study is composed of four main components covering the city of

321 N’Djaména, Chad i): An ongoing passive dog rabies surveillance system. Suspected rabid dogs

322 (dead or alive) were brought to the rabies diagnostic laboratory. No active collection of 
323 suspected dogs was done. For every rabies suspected dog, information on exposed humans was

324 collected on a routine basis. ii): A dog rabies mass vaccination campaign was done from October

325 to December 2012 and 2013 (23) Blood was taken from 104 dogs in 2012, prior to the start of

326 the mass vaccination campaign to assess the proportion of existing vaccination antibodies. The

327 Fluorescent antibody virus neutralization test (FAVN) was used for this purpose (33). Data on

328 rabid dogs and exposed humans was collected up to the end of 2015 for this paper. iii) A

329 mathematical transmission model of dog to dog and dog to human rabies transmission was

330 parametrized from the weekly number of rabid dogs and exposed humans collected under i)

331 prior, during and after the mass vaccination campaign (ii). iv) A phylogenetic and phylo-dynamic

332 analysis of the rabies virus strains collected under i) was used to assess their genetic closeness

333 and to estimate the basic reproductive number of the rabies transmission in dogs independently

334 from the mathematical transmission model (iii).

\section{Surveillance of dog rabies and human exposure}

336 Passive routine dog rabies surveillance started on 4 June 2012 and is currently ongoing in

337 N'Djaména at the Institut de Recherches en Elevage pour le Développement by standard

338 immunofluorescence as described in $(14,18)$. Prior to the mass vaccination campaign, the

339 average weekly incidence of dog rabies was of 0.33 dogs per 10,000 . For every laboratory-

340 confirmed rabid dog, on average 1.6 humans were reported to be exposed (from questioning the

341 dog owner) leading to a weekly incidence of 0.11 per 100,000 people. 
344 A citywide mass dog vaccination campaign including all 10 districts of N'Djaména took place in

3452012 and was repeated in 2013. In both campaigns, the objective was to vaccinate $70 \%$ of the

346 total dog population of N'Djaména with the dog rabies vaccine Rabisin ${ }^{\mathrm{TM}}$ (Merial Inc. Lyon,

347 France). The vaccination campaigns began in the first week of October 2012 and 2013 and lasted

348 for a total of 13 weeks until the first week of January of the next year. Vaccination took place

349 only on Friday to Sunday due to availability of staff and participation of the public during these

350 days (as evaluated in previous studies) (6). Every Friday to Sunday, ten fixed post vaccination

351 teams were set up in one of 12 (13 in 2013) areas of the city corresponding to administrative

352 boundaries. Over the three day period, these teams vaccinated on average 1,433 (min. 24; max.

353 6,460) dogs in 2012 and 1,709 dogs (min. 67; max. 4,591) dogs in 2013, depending on the socio-

354 cultural and ecological context of the city district (Table 1). Details of the operational

355 performance and the results of the vaccination campaigns are published elsewhere (23) .

\section{Coverage assessment}

357 A coverage assessment was carried out each week after vaccination in the previously vaccinated

358 area Fig. S4. Vaccination zones and their analysis perimeter corresponded in most cases to a

359 district. The coverage assessment was composed of a household survey in randomly selected

360 geographical locations within the analysis perimeter to estimate the proportion of owned

361 vaccinated dogs. In addition, random transects were carried out with a car in the same zone to

362 estimate the dog density in the street and the proportion of ownerless dogs. Data from both

363 studies were then combined in one Bayesian statistical model as reported elsewhere (23).

364 Description of mathematical model of dog-dog and dog-human

365 transmission 
366 We use a deterministic population based model of ordinary differential equations extended from a

367 previously published model for dog to dog rabies transmission (14),

$$
\begin{aligned}
\frac{d S_{d}(t)}{d t} & =b_{d} N_{d}(t)+\lambda_{d} V_{d}(t)-r_{d} \beta_{d d} S_{d}(t) I_{d}(t)-\left(v_{d} \alpha_{d}(t)+m_{d}+\gamma_{d} N_{d}(t)\right) S_{d}(t), \\
\frac{d E_{d}(t)}{d t} & =r_{d} \beta_{d d} S_{d}(t) I_{d}(t)-\left(\sigma_{d}+v_{d} \alpha_{d}(t)+m_{d}+\gamma_{d} N_{d}(t)\right) E_{d}(t) \\
\frac{d I_{d}(t)}{d t} & =\sigma_{d} E_{d}(t)-\left(\mu_{d}+m_{d}+\gamma_{d} N_{d}(t)\right) I_{d}(t) \\
\frac{d V_{d}(t)}{d t} & =v_{d} \alpha_{d}(t)\left(S_{d}(t)+E_{d}(t)\right)-\left(\lambda_{d}+m_{d}+\gamma_{d} N_{d}(t)\right) V_{d}(t)
\end{aligned}
$$

368 where the state variables and parameters are defined in Tables S1 and S2 respectively. The total

369 dog population size is,

$$
N_{d}(t)=S_{d}(t)+E_{d}(t)+I_{d}(t)+V_{d}(t),
$$

370 and the density dependent death rate is,

$$
\gamma_{\mathrm{d}}=\frac{\mathrm{b}_{\mathrm{d}}-\mathrm{m}_{\mathrm{d}}}{\mathrm{K}_{\mathrm{d}}}
$$

371 where $K_{d}$ is described in Table $S 2$ and $b_{d}$ is required to be greater than $m_{d}$. We note here that we 372 assume density-dependent transmission and that in general, (1) is a non-autonomous model where $373 \alpha_{d}(t)$ varies with time,

$$
\alpha_{d}(t)=\alpha_{d}^{*}+\alpha_{0}^{(i)}(t)+\alpha_{1}^{(i)}(t) e^{-\varphi t},
$$

374 where $\alpha_{\mathrm{d}}^{*}$ is the (assumed) constant background vaccination rate, $\alpha_{0}^{(\mathrm{i})}(\mathrm{t})$ and $\alpha_{1}^{(\mathrm{i})}(\mathrm{t})$ are

375 campaign-dependent vaccination values for the $\mathrm{i}^{\text {th }}$ week, and $\varphi$ is a saturation parameter. Outside

376 of the campaigns, $\alpha_{d}(t)=\alpha_{d}^{*}$. We further restrict the values of $\alpha_{0}^{(i)}$ and $\alpha_{1}^{(i)}$ to ensure that $\alpha_{d}(t)$

377 is continuous so that the system for rabies transmission (1) has a unique solution that exists for all 378 time.

379 We similarly use an ordinary differential equation model for dog to human transmission based on $380(14)$ 


$$
\begin{aligned}
& \frac{d S_{h}(t)}{d t}=b_{h} N_{h}(t)-\beta_{h d} S_{h}(t) I_{d}(t)+a_{h} E_{h}(t)-m_{h} S_{h}(t), \\
& \frac{d E_{h}(t)}{d t}=\beta_{h d} S_{h}(t) I_{d}(t)-\left(a_{h}+\sigma_{h}+m_{h}\right) E_{h}(t), \\
& \frac{d I_{h}(t)}{d t}=\sigma_{h} E_{h}(t)-\left(m_{h}+\mu_{h}\right) I_{h}(t),
\end{aligned}
$$

381 where the total human population size is,

$$
N_{h}(t)=S_{h}(t)+E_{h}(t)+I_{h}(t)
$$

$382 \sigma_{\mathrm{h}}$ is the rate of progression from the exposed to the infectious state depending on the site of the 383 bite,

$$
\sigma_{\mathrm{h}}=\frac{\mathrm{P}_{2} \mathrm{P}_{6}}{\mathrm{i}_{\text {head }}}+\frac{\mathrm{P}_{3} \mathrm{P}_{7}}{\mathrm{i}_{\text {arm }}}+\frac{\mathrm{P}_{4} \mathrm{P}_{8}}{\mathrm{i}_{\text {trunc }}}+\frac{\mathrm{P}_{5} \mathrm{P}_{9}}{\mathrm{i}_{\text {leg }}}
$$

$384 \mathrm{a}_{\mathrm{h}}$ is the abortive rate of progression from the exposed back to the susceptible state,

$$
a_{h}=\frac{P_{2}\left(1-P_{6}\right)}{i_{\text {head }}}+\frac{P_{3}\left(1-P_{7}\right)}{i_{\text {arm }}}+\frac{P_{4}\left(1-P_{8}\right)}{i_{\text {trunc }}}+\frac{P_{5}\left(1-P_{9}\right)}{i_{\text {leg }}} \text {, }
$$

385 and the probabilities of biting different parts of the body $\left(\mathrm{P}_{2}\right.$ through $\left.\mathrm{P}_{5}\right)$, the probabilities of

386 subsequent progression to rabies $\left(\mathrm{P}_{6}\right.$ through $\left.\mathrm{P}_{9}\right)$, and the average time to do so $\left(1 / \mathrm{i}_{\xi}\right.$ where $\xi$ is

387 head, arm, trunk or leg), are described in more detail in the previous formulation of the model

388 (14). Fig. S5 shows a schematic of the model system. We note that the dynamics for rabies

389 transmission in humans is dependent on rabies transmission in dogs but the transmission in dogs

390 is independent of transmission in humans.

\section{Mathematical analysis}

393 In the absence of vaccination campaigns $\left(\alpha_{d}(t)=\alpha_{d}^{*}\right)$, the autonomous mathematical model for

394 rabies transmission in dogs (1) has a trivial disease-free equilibrium point, 


$$
\begin{aligned}
S_{d} & =\frac{\left(b_{d}+\lambda_{d}\right) K_{d}}{b_{d}+\lambda_{d}+v_{d} \alpha_{d}^{*}}, \\
E_{d} & =0, \\
I_{d} & =0, \\
V_{d} & =\frac{v_{d} \alpha_{d}^{*} K_{d}}{b_{d}+\lambda_{d}+v_{d} \alpha_{d}^{*}} .
\end{aligned}
$$

395 The control reproductive number for the dog rabies model is the number of dogs that one newly

396 introduced rabid dog would infect, assuming no disease in the population (with only background

397 vaccination),

$$
R_{c}=\frac{r_{d} \beta_{d d} \sigma_{d} K_{d}}{\left(\sigma_{d}+v_{d} \alpha_{d}^{*}+b_{d}\right)\left(\mu_{d}+b_{d}\right)}
$$

398 We omit the mathematical details here but can show that with only background vaccination (no

399 vaccination campaigns) when $R_{c}<1$, the disease-free equilibrium point (9) is locally

400 asymptotically stable and when $R_{c}>1$, the disease-free equilibrium point is unstable and there

401 exists a locally asymptotically stable endemic equilibrium point where rabies persists in the

402 population. Additionally, if there is no background vaccination, the control reproductive number

403 reduces to the basic reproductive number,

$$
\mathrm{R}_{0}=\frac{\mathrm{r}_{\mathrm{d}} \beta_{\mathrm{dd}} \sigma_{\mathrm{d}} \mathrm{K}_{\mathrm{d}}}{\left(\sigma_{\mathrm{d}}+\mathrm{b}_{\mathrm{d}}\right)\left(\mu_{\mathrm{d}}+\mathrm{b}_{\mathrm{d}}\right)}
$$

404 At any time, $t$, allowing for vaccination campaigns, the effective reproductive number, $\mathrm{R}_{\mathrm{e}}(\mathrm{t})$, 405 represents the expected number of new infections caused by one infectious dog,

$$
R_{e}(t)=\frac{r_{d} \beta_{d d} \sigma_{d} S_{d}(t)}{\left(\sigma_{d}+v_{d} \alpha_{d}(t)+m_{d}+\gamma_{d} N_{d}(t)\right)\left(\mu_{d}+m_{d}+\gamma_{d} N_{d}(t)\right)} .
$$

407

408 If we assume that the total dog population is at carrying capacity (which is reasonable because 409 the density of rabid dogs is low so has a minimal impact on the population density of dogs), the 410 effective reproductive number simplifies to, 
$411 \quad \mathrm{R}_{\mathrm{e}}(\mathrm{t})=\frac{\mathrm{r}_{\mathrm{d}} \beta_{\mathrm{dd}} \sigma_{\mathrm{d}} \mathrm{s}_{\mathrm{d}}(\mathrm{t})}{\left(\sigma_{\mathrm{d}}+v_{\mathrm{d}} \alpha_{\mathrm{d}}(\mathrm{t})+\mathrm{b}_{\mathrm{d}}\right)\left(\mu_{\mathrm{d}}+\mathrm{b}_{\mathrm{d}}\right)}$

412 The threshold density of susceptible dogs at which transmission occurs, $\mathrm{S}_{\mathrm{d}}^{*}$ is the density at which

$413 \mathrm{R}_{\mathrm{e}}=1$. From (12), outside of vaccination campaigns this is,

$414 \quad S_{d}^{*}=\frac{\left(\sigma_{d}+v_{d} \alpha_{d}^{*}+b_{d}\right)\left(\mu_{d}+b_{d}\right)}{r_{d} \beta_{d d} \sigma_{d}}$.

415 The threshold vaccination coverage reached in a campaign to eliminate transmission, $\psi^{*}$, is given

416 by,

$$
\psi^{*}=1-\frac{1}{\mathrm{R}_{\mathrm{c}}}
$$

417 when background vaccination takes place outside the campaign. Equivalently this is,

$$
\psi^{*}=\frac{K_{d}-S_{d}^{*}}{K_{d}}
$$

418 After the vaccination campaigns, the coverage of protected dogs decreases exponentially due to

419 population loss of susceptible dogs (proportionally $b_{d} /\left(\lambda_{d}+b_{d}\right): 57 \%$ for parameter values in

420 table S2) and due to loss of vaccine efficacy (proportionally $\lambda_{d} /\left(\lambda_{d}+b_{d}\right): 43 \%$ for parameter

421 values in table $\mathrm{S} 2$ ).

\section{Parameter estimation}

423 The values for most parameters are taken from the previous model (14) except where new 424 published results or new data have allowed for revised values. The parameter values and their 425 sources are summarised in table S2. The birth and death rates of dogs were calculated as in 426 previous work but the carrying capacity of dogs was revised to reflect a total population of $42725,103 \mathrm{dogs}$ in an area of $240 \mathrm{~km}^{2}$ as estimated in the 2012 coverage assessment. The vaccination 428 rate of dogs and the transmission rates from dogs to dogs and dogs to humans were estimated as 429 described below. 


\section{Dog vaccination rate}

432 The baseline study found that $12 \%(n=105)$ of all owned dogs had antibodies (and so could be

433 considered effectively vaccinated), implying that there was some ongoing background

434 vaccination outside of the two campaigns conducted in 2012 and 2013. The coverage assessment

435 estimated that for every 10 owned dogs, there was one unowned dog. Assuming that the

436 background vaccination rate was constant and the proportion of vaccinated dogs was at

437 equilibrium (9), with demographic and other vaccination parameters as in table S2, the per capita

438 background vaccination rate was $2.96 \times 10^{-3} /$ week.

439 The number of dogs marked as vaccinated in each campaign is shown in Table 1. We estimated

440 the vaccination rate parameters, $\alpha_{0}^{(\mathrm{i})}$ and $\alpha_{1}^{(\mathrm{i})}$ using a simple model of vaccination for each

441 campaign,

$$
\begin{aligned}
\frac{d U^{(i)}}{d t} & =b_{d}\left(K_{d}-U^{(i)}\right)-\bar{\alpha}_{d}^{(i)}(t) U^{(i)}, \\
\frac{d V^{(i)}}{d t} & =\bar{\alpha}_{d}^{(i)}(t) U^{(i)},
\end{aligned}
$$

442 where $\mathrm{U}^{(\mathrm{i})}$ is the density of all unmarked dogs, $\mathrm{V}^{(\mathrm{i})}$ is the density of dogs marked in campaign

443 week $i$, and $\bar{\alpha}_{d}^{(i)}(t)$ is the rate of marking dogs during campaign week i. We define time, $t$, as

444 varying from 0 at the start of each campaign week to 1 at the end of each campaign week.

445 The coverage assessment could only determine whether dogs were marked as vaccinated or not

446 and did not determine the immune status of dogs. We therefore ignore the efficacy of the

447 vaccination and do not consider background vaccination because these dogs would not be marked 448 as campaign-vaccinated dogs, so

$$
\bar{\alpha}_{d}^{(i)}(t)=\alpha_{0}^{(i)}(t)+\alpha_{1}^{(i)}(t) e^{-\varphi t}
$$


449 For simplicity we ignore rabies virus transmission, assume the dog population is at carrying

450 capacity, and ignore the death of marked dogs or the loss of marking collars during the campaign

451 week. Since the coverage assessment was conducted within three days of the vaccination

452 campaign, these assumptions are reasonable. We assume that the markings from the 2012

453 campaign do not last until 2013 so for both campaigns, the initial density of unmarked dogs is

454 equal to the carrying capacity,

$455 \mathrm{U}^{(1)}(0)=\mathrm{K}_{\mathrm{d}}$,

$456 \quad(18 a)$

457 and from continuity,

$458 \quad \mathrm{U}^{(\mathrm{i})}(0)=\mathrm{U}^{(\mathrm{i}-1)}(1) \quad$ for $\mathrm{i}>1$.

459 The initial density of dogs marked during a campaign week is zero,

$$
\mathrm{V}^{(\mathrm{i})}(0)=0 \quad \text { for } \mathrm{i} \geq 1 \text {. }
$$

460 We fix $\varphi=100$. To ensure that $\alpha_{d}(t)$ is continuous, we set

$$
\begin{array}{ll}
\alpha_{0}^{(1)}+\alpha_{1}^{(1)} & =0 \\
\alpha_{0}^{(i)}+\alpha_{1}^{(i)} & =\alpha_{0}^{(i-1)}+\alpha_{1}^{(i-1)} e^{-\varphi} \quad \text { for } \mathrm{i}>1 .
\end{array}
$$

461 The final density of dogs marked in a campaign week, $\mathrm{V}^{(\mathrm{i})}(1)$ is set equal to the number of

462 marked dogs estimated from the coverage assessment for that week (Table 1) divided by the

463 campaign area for that year $\left(240 \mathrm{~km}^{2}\right.$ in 2012 and $285 \mathrm{~km}^{2}$ in 2013). Condition (19) and the

464 ordinary differential equations for the vaccination model (16) with its boundary conditions

465 provide two sets of equations for each campaign week. For other parameter values as provided in

466 table S2, we numerically simulate the vaccination model using an adaptive step-size Runge-Kutta

467 method (ode45) and then use a root-finding algorithm (fzero) to calculate $\alpha_{0}^{(\mathrm{i})}$ and $\alpha_{1}^{(\mathrm{i})}$ (in

468 MATLAB, version 8.5) for each campaign week. Fig. S6 shows the final estimated vaccination 469 rates during the two campaigns. 


\section{Rate of loss of vaccine immunity}

472 In 2012, before the vaccination campaigns were conducted, a total of 105 dogs in N'Djaména

473 were tested for antibody titers, vaccinated and then followed up over a period of one year. Of

474 these dogs, 58 had initial antibody titers that showed no previous vaccination and were

475 successfully followed up over the entire year. After one year, $44 \mathrm{dogs}$ had antibody titers above

$4760.5 \mathrm{IU}$, which, as a conservative estimate, we considered protective (34). We calculated the rate

477 of loss of vaccine decay, $\lambda_{d}$, assuming exponential decay and a relative value of 0.76 after 52

478 weeks.

\section{Rabies transmission rates}

480 The number of rabid dogs and exposed humans recorded per week since 4 June 2012 are shown

481 in the additional file RabiesData1.txt. Recording of both human and dog cases is ongoing but the 482 analysis only included cases until the end of October 2015. We divide the numbers of dogs and

483 humans by the area estimated in the coverage assessment of the 2012 vaccination campaign (240 $484 \mathrm{~km}^{2}$ ) to provide the densities of rabid dogs and exposed humans.

485 We first fit the dog to dog transmission rate, $\beta_{\mathrm{dd}}$, for the model with transmission only in dogs

486 (1) with the data for the number of rabid dogs with other parameter values as described in

487 table S2 and the vaccination rate as described above. We then use this value for $\beta_{\text {dd }}$ to estimate 488 the dog to human transmission rate, $\beta_{\mathrm{hd}}$, for the full model with transmission between dogs (1)

489 and to humans (5) with the data for number of exposed humans.

490 To fit $\beta_{\mathrm{dd}}$, we numerically simulate (1) using an adaptive step-size Runge-Kutta method and

491 minimise the Euclidean distance between the simulated incidence of infectious dogs (from the 492 first term of the right hand side of (1c)), and the observed weekly incidence of infectious dogs in 
493 MATLAB. We assume that the probability of detecting a rabid dog, $\mathrm{p}_{\mathrm{d}}=0.5$ so that on average

494 there were twice as many rabid dogs as those detected. There is little data on this parameter but

495 our sensitivity analysis showed that unless $p_{d}$ is very low, the estimated values for $\beta_{d d}$ did not

496 change much (Fig. S3). We assume that the initial condition for the ordinary differential

497 equations in June 2012 is at the unique endemic equilibrium (with $\alpha_{d}(t)=\alpha_{d}^{*}$ ) and the dog

498 density is at carrying capacity.

499 We similarly fit $\beta_{\mathrm{hd}}$ by numerically simulating (1) and (5) and minimising the Euclidean distance

500 between the simulated density of exposed humans, $E_{h}(t)$, and the observed density of exposed

501 humans on a weekly time step in MATLAB. Here we assume perfect detection of exposed

502 humans $\left(p_{h}=1\right)$ and that the initial condition in June 2012 is at the unique endemic equilibrium

503 with a population density of humans of 4833 humans $/ \mathrm{km}^{2}$ (from a total population size of 1.16

504 million in 2012 estimated from the 2011 population size of 1.079 million using a growth rate of

$5057.5 \%)(35,36)$.

506

\section{Phylogenetic importation analysis}

508 To investigate the hypothesis of a reintroduction of dog rabies virus in N'Djamena from outside

509 of the city after the vaccination campaigns, we performed a phylogenetic analysis using the

510 previously described 29 complete nucleoprotein sequences of rabies virus isolates collected

511 between August 2011 and January 2014, with the inclusion of one supplementary sequence of a

512 isolate collected during this period (GenBank accession number KY124541), in addition to the

513 sequences of the three first isolates collected in the city after this period (from February 2014 to

514 January 2015, GenBank accession numbers MF538629-31) and to published available sequences

515 from Chad $(n=1)$ and from neighboring countries $(n=14)$. Using jModelTest2 $(37,38)$, the best-fit

516 model of nucleotide substitution according to the Bayesian Information Criterion was the general 
517 time reversible model with proportion of invariable sites plus gamma-distributed rate

518 heterogeneity $(\mathrm{GTR}+\mathrm{I}+\Gamma 4)$. A phylogenetic tree was then estimated using the maximum

519 likelihood (ML) method available in PhyML 3.0 (39) utilizing SPR branch-swapping. The

520 robustness of individual nodes on the phylogeny was estimated using 1,000 bootstrap replicates

521 and using the approximate likelihood ratio test (aLRT) with SH-like supports (40).

\section{Sensitivity analysis}

524 We conducted local and global sensitivity analysis of the control reproductive number, $\mathrm{R}_{\mathrm{c}},(10)$

525 to the model parameters (Fig. S7). We used the normalized forward sensitivity index for the local

526 analysis $(41,42)$ at the parameter values defined in table S2 and the partial rank correlation

527 coefficients for the global analysis (43), assuming all parameters were uniformly distributed in

528 the intervals: $r_{d} \in[0.049,1], \beta_{\mathrm{dd}} \in[0.00292,0.0614], \mathrm{K}_{\mathrm{d}} \in[10.5,221], \sigma_{\mathrm{d}} \in$

$529[0.0239,0.504], b_{d} \in[0.0013,0.0273], \mu_{d} \in[0.123,2.59], v_{d} \in[0.094,1]$ and $\alpha_{d}^{*} \in$

$530 \quad[0.000296,0.00622]$. Both the local and global analysis showed that the probability of

531 developing rabies, $r_{d}$, the transmission rate, $\beta_{d d}$, the carrying capacity, $K_{d}$ and the rabies virus

532 induced mortality rate, $\mu_{\mathrm{d}}$, had a strong impact on the threshold for sustained transmission, $\mathrm{R}_{\mathrm{c}}$,

533 while the other parameters had minimal impact.

534

535

\section{Stochastic model simulations}

537 We derived and numerically simulated a stochastic dog to dog transmission model based on (1), 538 with the master equation, 


$$
\frac{\mathrm{dP}(\mathrm{n}, \mathrm{t})}{\mathrm{dt}}=\sum_{i}\left[\mathrm{~W}_{\mathrm{i}}\left(\mathrm{n} \mid \mathrm{m}_{\mathrm{i}}\right) \mathrm{P}\left(\mathrm{m}_{\mathrm{i}}, \mathrm{t}\right)-\mathrm{W}_{\mathrm{i}}\left(\mathrm{m}_{\mathrm{i}} \mid \mathrm{n}\right) \mathrm{P}(\mathrm{n}, \mathrm{t})\right]
$$

539 where $\mathrm{n}$ is any state of the system at time $\mathrm{t}$ and $\mathrm{W}_{\mathrm{i}}$ are the transmission rates deduced from the

540 parameters in table S2, using the Gillespie algorithm with the tau-leaping simulation method (44,

$54145)$. Fig. S8 shows a sample stochastic simulation of the density of exposed and infectious dogs

542 with the corresponding simulation of the deterministic model and the underlying data for the

543 number of infectious dogs. Fig. 3B shows that the mean of 500 simulation runs of the stochastic

544 model declines after the first vaccination campaign in a similar manner to the deterministic

545 model.

\section{Phylodynamic analysis}

54729 sequences of canine rabies viruses, collected between August 2011 and June 2013, were

548 analysed with Beast v2 (25). We chose a Hasegawa-Kishino-Yano (HKY) model for substitutions

549 with a relaxed log-normal clock (46). We assumed an exponential (0.001) prior for the mean

550 rate, an exponential $(0.3333)$ prior for the standard deviation, and a log-normal $(1,1.25)$ prior

551 for kappa.

552 For the epidemiological model, we chose the birth-death skyline model (47). We used a log

553 normal $(0,1)$ prior for the effective reproductive number, $R_{e}$, and allowed $R_{e}$ to change in

554 January 2013, August 2012, and April 2012, i.e. every 4.8 months prior to the last sample in June

555 2013. We assumed a uniform prior on the interval $(9.44,9.5)$ for the dog removal rate

556 (corresponding to an expected infection time of exposed and active rabies between (1/9.5,1/

557 9.44) years, which is about 1.1 months. The sampling probability of a rabid dog was assumed to

558 be 0 prior to the first sample, and uniform on $(0.4,0.6)$ between the first and last sample. The

559 time of the initial case in that transmission chain was assumed to be a uniform prior on $(0,20)$, 
560 prior to the most recent sample. We ran the Markov chain Monte Carlo (MCMC) simulations for

$56110^{9}$ steps. We neglected the first $10 \%$ of the states as a burn-in period. The effective sample size

562 of all parameters was 350 or higher, implying that we obtained substantial mixing.

563 In order to investigate sensitivity towards our assumption of a constant sampling proportion, we

564 performed a second analysis allowing the sampling proportion to change at the same time points

565 as when the $\mathrm{R}_{\mathrm{e}}$ changes. As above, sampling was assumed to be 0 prior to the oldest sample.

566 Further sampling was assumed to be uniform on $(0.2,0.6)$ in each interval (compared to uniform

567 on $(0.4,0.6)$ above. As shown in fig. S9 the results do not change qualitatively.

568

569 Limitations of the study

570 The study is limited by the low number of rabies viruses that were isolated during the rabies mass

571 vaccination campaign. For this reason, the credibility intervals of the phylodynamic estimation of

572 the basic reproductive number are wide. Another limitation is that we could not clearly identify

573 the source of the rabies viruses that were re-introduced from outside the city. Further ongoing

574 research will relate the rabies viruses collected in N'Djamena in this study with strains that will

575 be collected countrywide.

\section{References and notes}

577 1. K. Hampson, J. Dushoff, J. Bingham, G. Bruckner, Y. H. Ali, A. Dobson, Synchronous cycles of

578

579

580

581 domestic dog rabies in sub-Saharan Africa and the impact of control efforts. Proc.Natl.Acad.Sci.U.S.A 104, 7717-7722 (2007).

2. K. Hampson, L. Coudeville, T. Lembo, M. Sambo, A. Kieffer, M. Attlan, J. Barrat, J. D. Blanton, D. J. Briggs, S. Cleaveland, P. Costa, C. M. Freuling, E. Hiby, L. Knopf, F. Leanes, F. X. Meslin, A. Metlin, M. E. Miranda, T. Muller, L. H. Nel, S. Recuenco, C. E. Rupprecht, C. Schumacher, L. Taylor, M. A. Vigilato, J. Zinsstag, J. Dushoff, P. Global Alliance for Rabies Control Partners for Rabies, Estimating the global burden of endemic canine rabies. PLoS Negl Trop Dis 9, e0003709 (2015); published online EpubApr (10.1371/journal.pntd.0003709).

3. S. Cleaveland, F. Lankester, S. Townsend, T. Lembo, K. Hampson, Rabies control and elimination: a test case for One Health. Vet Rec 175, 188-193 (2014); published online EpubAug 30 (10.1136/vr.g4996). 
4. K. Hampson, J. Dushoff, S. Cleaveland, D. T. Haydon, M. Kaare, C. Packer, A. Dobson, Transmission dynamics and prospects for the elimination of canine rabies. PLoS biology 7, e53 (2009); published online EpubMar 10 (10.1371/journal.pbio.1000053).

5. J. Zinsstag, Towards a science of rabies elimination. Infectious diseases of poverty 2, 22 (2013)10.1186/2049-9957-2-22).

6. U. Kayali, R. Mindekem, N. Yemadji, P. Vounatsou, Y. Kaninga, A. G. Ndoutamia, J. Zinsstag, Coverage of pilot parenteral vaccination campaign against canine rabies in N'Djamena, Chad. Bull. World Health Organ 81, 739-744 (2003).

7. B. Obrist, N. Iteba, C. Lengeler, A. Makemba, C. Mshana, R. Nathan, S. Alba, A. Dillip, M. W. Hetzel, I. Mayumana, A. Schulze, H. Mshinda, in PLoS Medicine. (2007), vol. 4, pp. 1584-1588.

8. S. Dürr, M. Meltzer, R. Mindekem, J. Zinsstag, Owner valuation of rabies vaccination in dogs, Chad. Emerging Infectious Diseases 14, 1650-1652 (2008).

9. C. Talbi, P. Lemey, M. A. Suchard, E. Abdelatif, M. Elharrak, N. Jalal, A. Faouzi, J. E. Echevarría, S. V. Morón, A. Rambaut, Phylodynamics and human-mediated dispersal of a zoonotic virus. PLoS pathogens 6, e1001166 (2010).

10. J. Zinsstag, E. Schelling, D. Waltner-Toews, M. Tanner, From "one medicine" to "one health" and systemic approaches to health and well-being. Prev.vet.med. 101, 148-156 (2011).

11. G. Hirsch Hadorn, H. Hoffmann-Reim, S. Biber-klemm, W. Grossenbacher, D. Joye, C. Pohl, U. Wiesmann, E. Zemp, Handbook of Transdisciplinary Research. G. Hirsch Hadorn, H. HoffmannReim, S. Biber-klemm, W. Grossenbacher, D. Joye, C. Pohl, U. Wiesmann, E. Zemp, Eds., (Springer, 2008), pp. 1-448.

12. D. F. Charron, Ecosystem approaches to health for a global sustainability agenda. EcoHealth 9 , 256-266 (2012); published online EpubSep (10.1007/s10393-012-0791-5).

13. S. E. Townsend, I. P. Sumantra, Pudjiatmoko, G. N. Bagus, E. Brum, S. Cleaveland, S. Crafter, A. P. Dewi, D. M. Dharma, J. Dushoff, J. Girardi, I. K. Gunata, E. F. Hiby, C. Kalalo, D. L. Knobel, I. W. Mardiana, A. A. Putra, L. Schoonman, H. Scott-Orr, M. Shand, I. W. Sukanadi, P. P. Suseno, D. T. Haydon, K. Hampson, Designing programs for eliminating canine rabies from islands: Bali, Indonesia as a case study. PLoS Negl Trop Dis 7, e2372 (2013)10.1371/journal.pntd.0002372).

14. J. Zinsstag, S. Dürr, M. A. Penny, R. Mindekem, F. Roth, S. M. Gonzalez, S. Naissengar, J. Hattendorf, Transmission dynamics and economics of rabies control in dogs and humans in an African city. Proceedings of the National Academy of Sciences of the United States of America 106, 14996-15001 (2009).

15. P. Klepac, C. J. E. Metcalf, A. R. McLean, K. Hampson, Towards the endgame and beyond: complexities and challenges for the elimination of infectious diseases. Philosophical Transactions of the Royal Society B: Biological Sciences 368, (2013); published online EpubAugust 5, 2013 (10.1098/rstb.2012.0137).

16. U. Kayali, R. Mindekem, G. Hutton, A. G. Ndoutamia, J. Zinsstag, Cost-description of a pilot parenteral vaccination campaign against rabies in dogs in N'Djamena, Chad. Tropical medicine \& international health : TM \& IH 11, 1058-1065 (2006).

17. T. Jibat, H. Hogeveen, M. C. Mourits, Review on dog rabies vaccination coverage in Africa: a question of dog accessibility or cost recovery? PLoS Negl Trop Dis 9, e0003447 (2015); published online EpubFeb (10.1371/journal.pntd.0003447).

18. U. Kayali, R. Mindekem, N. Yemadji, A. Oussiguere, S. Naissengar, A. G. Ndoutamia, J. Zinsstag, Incidence of canine rabies in N'Djamena, Chad. Prev.vet.med. 61, 227-233 (2003).

19. S. Durr, S. Naissengar, R. Mindekem, C. Diguimbye, M. Niezgoda, I. Kuzmin, C. E. Rupprecht, J. Zinsstag, Rabies diagnosis for developing countries. PLoS.Negl.Trop.Dis. 2, e206 (2008).

20. R. Mindekem, U. Kayali, N. Yemadji, A. G. Ndoutamia, J. Zinsstag, [Impact of canine demography on rabies transmission in N'djamena, Chad]. $M$, decine tropicale : revue du Corps de sant, colonial 65, 53-58 (2005). 
638

639

640

641

642

643

644

645

646

647

648

649

650

651

652

653

654

655

656

657

658

659

660

661

662

663

664

665

666

667

668

669

670

671

672

673

674

675

676

677

678

679

680

681

682

683

684

685

686

21. S. Durr, R. Mindekem, Y. Kaninga, D. Doumagoum Moto, M. I. Meltzer, P. Vounatsou, J. Zinsstag, Effectiveness of dog rabies vaccination programmes: comparison of owner-charged and free vaccination campaigns. Epidemiol Infect 137, 1558-1567 (2009); published online EpubNov (10.1017/S0950268809002386).

22. J. Frey, R. Mindekem, H. Kessely, D. Doumagoum Moto, S. Naissengar, J. Zinsstag, E. Schelling, Survey of animal bite injuries and their management for an estimate of human rabies deaths in N'Djamena, Chad. Tropical medicine \& international health : TM \& IH 18, 1555-1562 (2013); published online EpubDec (10.1111/tmi.12202).

23. M. Lechenne, A. Oussiguere, K. Naissengar, R. Mindekem, L. Mosimann, G. Rives, J. Hattendorf, D. D. Moto, I. O. Alfaroukh, J. Zinsstag, T. Comm, Operational performance and analysis of two rabies vaccination campaigns in N'Djamena, Chad. Vaccine 34, 571-577 (2016); published online EpubJAN 202016 (10.1016/j.vaccine.2015.11.033).

24. C. Talbi, E. C. Holmes, P. de Benedictis, O. Faye, E. Nakoune, D. Gamatie, A. Diarra, B. O. Elmamy, A. Sow, E. V. Adjogoua, O. Sangare, W. G. Dundon, I. Capua, A. A. Sall, H. Bourhy, Evolutionary history and dynamics of dog rabies virus in western and central Africa. J Gen Virol 90, 783-791 (2009); published online EpubApr (10.1099/vir.0.007765-0).

25. R. Bouckaert, J. Heled, D. Kuhnert, T. Vaughan, C. H. Wu, D. Xie, M. A. Suchard, A. Rambaut, A. J. Drummond, BEAST 2: a software platform for Bayesian evolutionary analysis. PLoS Comput Biol 10, e1003537 (2014); published online EpubApr (10.1371/journal.pcbi.1003537).

26. H. Bourhy, E. Nakoune, M. Hall, P. Nouvellet, A. Lepelletier, C. Talbi, L. Watier, E. C. Holmes, S. Cauchemez, P. Lemey, C. A. Donnelly, A. Rambaut, Revealing the Micro-scale Signature of Endemic Zoonotic Disease Transmission in an African Urban Setting. PLoS Pathog 12, e1005525 (2016); published online EpubApr (10.1371/journal.ppat.1005525).

27. F. Anyiam, M. Lechenne, R. Mindekem, A. Oussigere, S. Naissengar, I. O. Alfaroukh, C. Mbilo, D. D. Moto, P. G. Coleman, N. Probst-Hensch, J. Zinsstag, Cost-estimate and proposal for a development impact bond for canine rabies elimination by mass vaccination in Chad. Acta tropica, (2016); published online EpubNov 23 (10.1016/j.actatropica.2016.11.005).

28. M. K. Morters, O. Restif, K. Hampson, S. Cleaveland, J. L. Wood, A. J. Conlan, Evidence-based control of canine rabies: a critical review of population density reduction. The Journal of animal ecology 82, 6-14 (2013); published online EpubJan (10.1111/j.1365-2656.2012.02033.x).

29. M. Begon, M. Bennett, R. G. Bowers, N. P. French, S. M. Hazel, J. Turner, A clarification of transmission terms in host-microparasite models: numbers, densities and areas. Epidemiol Infect 129, 147-153 (2002); published online EpubAug (

30. M. Lechenne, K. Naissengar, A. Lepelletier, I. O. Alfaroukh, H. Bourhy, J. Zinsstag, L. Dacheux, Validation of a Rapid Rabies Diagnostic Tool for Field Surveillance in Developing Countries. PLoS Negl Trop Dis 10, e0005010 (2016); published online EpubOct (10.1371/journal.pntd.0005010).

31. D. N. Durrheim, H. Rees, D. J. Briggs, L. H. Blumberg, Mass vaccination of dogs, control of canine populations and post-exposure vaccination--necessary but not sufficient for achieving childhood rabies elimination. Tropical medicine \& international health : TM \& IH 20, 682-684 (2015); published online EpubJun (10.1111/tmi.12474).

32. R. Mindekem, M. S. Lechenne, K. S. Naissengar, A. Oussiguere, B. Kebkiba, D. D. Moto, I. O. Alfaroukh, L. T. Ouedraogo, S. Salifou, J. Zinsstag, Cost Description and Comparative Cost Efficiency of Post-Exposure Prophylaxis and Canine Mass Vaccination against Rabies in N'Djamena, Chad. Front Vet Sci 4, 38 (2017)10.3389/fvets.2017.00038).

33. F. Cliquet, M. Aubert, L. Sagne, Development of a fluorescent antibody virus neutralisation test (FAVN test) for the quantitation of rabies-neutralising antibody. J Immunol Methods 212, 79-87 (1998); published online EpubMar 1 (S0022175997002123 [pii]).

34. S. M. Moore, C. A. Hanlon, Rabies-specific antibodies: measuring surrogates of protection against a fatal disease. PLoS Negl Trop Dis 4, e595 (2010)10.1371/journal.pntd.0000595). 
687

688

689

690

691

692

693

694

695

696

697

698

699

700

701

702

703

704

705

706

707

708

709

710

711

712

713

714

715

716

717

718

\section{Acknowledgements:}

720 We thank all the community leaders and vaccinators in N'Djaména. We thank Thomas Smith for

721 helpful comments and discussions, and anonymous reviewers for helpful comments and

722 suggestions. There is no paid or unpaid consulting and there are no patents related to this work.

35. Indexmundi. (2015).

36. Deuxième recensement général de la population ed de l'habitat (2009).

37. D. Darriba, G. L. Taboada, R. Doallo, D. Posada, jModelTest 2: more models, new heuristics and parallel computing. Nature methods 9, 772 (2012); published online EpubJul 30 (10.1038/nmeth.2109).

38. S. Guindon, O. Gascuel, A simple, fast, and accurate algorithm to estimate large phylogenies by maximum likelihood. Syst Biol 52, 696-704 (2003); published online EpubOct (

39. S. Guindon, J. F. Dufayard, V. Lefort, M. Anisimova, W. Hordijk, O. Gascuel, New algorithms and methods to estimate maximum-likelihood phylogenies: assessing the performance of PhyML 3.0. Syst Biol 59, 307-321 (2010); published online EpubMay (10.1093/sysbio/syq010).

40. M. Anisimova, O. Gascuel, Approximate likelihood-ratio test for branches: A fast, accurate, and powerful alternative. Syst Biol 55, 539-552 (2006); published online EpubAug (10.1080/10635150600755453).

41. N. Chitnis, J. M. Hyman, J. M. Cushing, Determining important parameters in the spread of malaria through the sensitivity analysis of a mathematical model. Bulletin of Mathematical Biology 70, 1272-1296 (2008).

42. L. H. Arriola, J.M.;, Mathematical and statistical estimation approaches in epidemiology. G. H. Chowell, J.M; Bettencourt, C.; Castillo-Chavez, C. eds., Ed., (Springer, Dordrecht, 2009).

43. J. Wu, R. Dhingra, M. Gambhir, J. V. Remais, Sensitivity analysis of infectious disease models: methods, advances and their application. Journal of the Royal Society Interface 10, 20121018 (2013).

44. D. T. Gillespie, Exact stochastic simulation of coupled chemical reactions. J. Phys. Chem. 81, 2340-2361 (1977)10.1021/j100540a008).

45. Y. G. Cao, D.T.; Petzold, R., Efficient step size selection of the tau-leaping simulation method. J. Phys. Chem. 124, 844109 (2006).

46. A. J. Drummond, S. Y. Ho, M. J. Phillips, A. Rambaut, Relaxed phylogenetics and dating with confidence. PLoS biology 4, e88 (2006); published online EpubMay (10.1371/journal.pbio.0040088).

47. T. Stadler, D. Kuhnert, S. Bonhoeffer, A. J. Drummond, Birth-death skyline plot reveals temporal changes of epidemic spread in HIV and hepatitis C virus (HCV). Proc Natl Acad Sci U S A 110, 228233 (2013); published online EpubJan 2 (10.1073/pnas.1207965110). 
725 Funding for this study was provided by the Chadian government, the UBS Optimus Foundation

726 and the Swiss Federal Food Safety and Veterinary Office (FFSVO), the European Union Seventh

727 Framework Programme PREDEMICS (grant \#278433), the Wolfermann-Nägeli Foundation, the

728 Emilia Guggenheim Schnurr Foundation and the Swiss National Science Foundation (grant\#:

729 310030_160067 / 1).

730 Support was provided by the UBS Optimus Foundation (M.Le, R.M.), the Swiss Federal Food

731 Safety and Veterinary Office (FFSVO) (M.Le.), the Wolfermann-Nägeli Foundation (M.Le.),

732 Swiss Nationals Science foundation grant number 310030-160067 (M. La.),. and the European

733 Research Council under the Seventh Framework Programme of the European Commission

734 (PhyPD: grant agreement number 335529)(T.S.).

735 Requests for rabies virus strains require a Material Transfer Agreement and should be sent to

736 Service Naissengar Institut de Recherches en Elevage pour le Développement, BP 433,

737 N'Djaména, Chad email : naissengar@gmail.com

738

739

740 Author contributions:

741 J.Z.: conceived the study, J.Z. and I. A. O. applied for funding. J.Z., M. Le, M. La. J.H., A. L., H.

742 B., L. D., T.S., N.C. wrote the manuscript. M.Le., M.La., R.M., S.N., A.O., G.R., J.T., S.M.,

743 M.O., Y.M., A.T. participated in the acquisition and analysis of data. J. Z., M.La. N.C.

744 contributed to the mathematical modelling. S. N. did all the rabies diagnostic work. A.O.

745 coordinated the rabies mass vaccination campaign. J.Z., M. Le, M. La. A.O., K. B., M.O.,

746 D.D.M., I.A.O., Y.M., A.T. J. H., A.L., H. B., T.S., provided a substantial intellectual

747 contribution. K.B., J.T. D.D.M., I.A.O. provided administrative, technical or supervisory 
748 support. D.D.M. took medical responsibility of the study A.L., L.K., H.B., L.D., participated in

749 the analysis of molecular genetic data of the rabies viruses. N.C.: Supervised the mathematical

750 modelling.

751 Competing interests:

752 None declared

753 Data and materials availability:

754 The dog and human rabies incidence data and the genetic sequences of 29 dog rabies virus N-

755 protein are available in the Supplementary materials. The N-protein sequences are deposited in

756 GenBank (https://www.ncbi.nlm.nih.gov/nuccore/) under the accession numbers KU564980-99

757 and KY124532-40.

758 - RabiesData1.txt (incidence data)

759 - RabiesData2.txt (genetic sequences of 29 dog rabies virus N-protein deposited in GenBank)

760

761 


\section{Tables}

763

764 Table 1. Number of dogs vaccinated in each week of the vaccination campaigns. The campaign

765 in 2012 started on 8 October 2012 (week 19) and in 2013 started on 30 September 2013 (week

766 70), as described in (23).

767

\begin{tabular}{|c|c|c|}
\hline Vaccination Week & Vaccinated dogs (2012) & Vaccinated dogs (2013) \\
\hline 1 & 834 & 722 \\
\hline 2 & 181 & 468 \\
\hline 3 & 376 & 330 \\
\hline 4 & 24 & 434 \\
\hline 5 & 793 & 67 \\
\hline 6 & 2901 & 1173 \\
\hline 7 & 6460 & 928 \\
\hline 8 & 1393 & 4215 \\
\hline 9 & 3074 & 4372 \\
\hline 10 & 1698 & 3424 \\
\hline 11 & 311 & 4591 \\
\hline 12 & 385 & 979 \\
\hline 13 & 209 & 525 \\
\hline
\end{tabular}




\section{Figures}

772 Fig. 1 (A) Cumulative incidence of dog rabies cases and human exposure. Cumulative incidence

773 of recorded cases of dog rabies (infectious dogs) and simulated incidence of dog rabies in

774 N'Djaména from 6 June 2012 to the end of October 2015. (B) Cumulative incidence of recorded

775 human exposure to rabid dogs and simulated incidence of human exposure to rabid dogs in

776 N'Djaména from 6 June 2012 to the end of October 2015.

778 Fig. 2 (A) Density of vaccinated and unvaccinated dogs in relation to the effective reproductive

779 number. Density of susceptible (blue lines) and vaccinated (red lines) dogs against time since 6

780 June 2012. The solid lines show the simulated values from an ordinary differential equation

781 transmission model from June 2012 to October 2015. (B) The effective reproductive number, $R_{e}$,

782 and vaccination coverage against time. The solid red line shows the vaccination coverage and the

783 solid blue line shows the effective reproductive number - both estimated from the ordinary

784 differential equation transmission model. The black line is the median $\mathrm{R}_{\mathrm{e}}$ obtained from the

785 phylogenetic sequencing data, with upper and lower 95\% credible intervals as black dashed lines.

786 Fig. 3 (A) Stochastic simulations of the interruption of transmission. Distribution of the simulated

787 expected date of interruption of transmission from 1000 simulation runs of the stochastic model

788 of dog rabies virus transmission. (B) Mean and $90 \%$ credible interval for exposed and infectious

789 dogs from 500 runs of the stochastic model.

790

791 Fig. 4: Phylogeny of rabies viruses isolated during and after the mass vaccination campaign.

792 Maximum likelihood phylogeny of nucleoprotein sequences from rabies virus isolates collected 
793 in Chad (from N'Djaména and other regions) from August 2011 to January 2015, and from

794 sequences of previous rabies virus isolates originated from Chad and from other neighboring

795 countries. Sequences in blue were obtained from isolates collected in N'Djaména, Chad, during

796 the period from August 2011 to January 2014, excepted for the sequences with an asterisk which

797 correspond to isolates collected outside of N'Djaména or without any precise origin (for one

798 isolate) during the same period. Sequences in red are those obtained from isolates collected in

799 N'Djaména from February 2014 to January 2015. Only bootstrap values $>70$ are indicated on

800 selected nodes. A scale indicating genetic distance is presented by the horizontal bar. The tree is

801 mid-point rooted for clarity only. GenBank accession numbers of published sequences used in

802 this tree are: EU853590 (07072RCA), EU853651 (07149RCA), EU038107 (35RD8005),

803 KT119773 (8801CAM), KX148243 (8803CAM), U22635 (8804CAM), EU853654 (9014NIG),

804 KX148208 (92029CAR), KT119779 (9218TCH), KT119784 (9502CAM), KF977826

805 (CAR_11_001h), KC196743 (DRV_NG11), EU038108 (RD128), EU038096 (RD137) and

806 EU038093 (RD9305HN).

807

808

809

810

811 